Objectives Women with cervical cancer who undergo radical hysterectomy are often treated postoperatively with chemoradiation. The patient selection that minimizes adjuvant treatment is valuable. We compared two methods for predicting postoperative adjuvant treatment of patients with stage IB2 cervical cancer.

Methods This multicenter retrospective study included 272 women with IB2 tumors. A receiver operating characteristic curve (ROC) analysis was used to determine the optimal tumor cutoff size to predict adjuvant treatment. A second analysis compared the rate of adjuvant treatment between women with and without lymph vascular space involvement (LVSI).

Results According to the ROC, the optimal cutoff value of tumor size for predicting adjuvant treatment was $2.95 \mathrm{~cm}$ (sensitivity 0.70 , specificity 0.67 ). Tumors were $\geq 3.0 \mathrm{~cm}$ in $166(61.0 \%)$ women. The rate of adjuvant treatment was higher in women with larger tumor diameter $(73.8 \%$ vs. $47.9 \%, \mathrm{p}<0.0001)$. of the 241 women with a LVSI record, LVSI was present in 81 (34\%) women. Among women with LVSI, rates were higher of positive lymph nodes $(41.0 \%$ vs $14.5 \%, \quad \mathrm{p}<0.0001)$ and postoperative adjuvant treatment $(83.3 \%$ vs. $53.7 \%, \mathrm{p}<0.001)$. Among women with tumor size $\geq 3.0 \mathrm{~cm}$ and LVSI, the rate of adjuvant treatment was $90.0 \%$. In the multivariate analysis, both tumor size $\geq 3.0 \mathrm{~cm}$ and the presence of LVSI were independently associated with adjuvant treatment (OR 3.9, 95\% CI 2.1-7.1; p <0.0001 and OR 4.9, 95\% CI 2.4-10.0; $\mathrm{p}<0.0001$, respectively).

Conclusions These data should be weighed in multidisciplinary consultation with radiation oncologists when deciding treatment strategy.

\section{EPV035/\#138 RADICAL TRACHELECTOMY. EXPERIENCE IN KAZIOR}

${ }^{1}$ A Satanova, ${ }^{2} \mathrm{D}$ Kaidarova*, ${ }^{3} \mathrm{R}$ Bolatbekova, ${ }^{1} \mathrm{Y}$ Kukubassov, ${ }^{1} \mathrm{D}$ Kaldybekov. ${ }^{1}$ Kazakh Institute of Oncology and Radiology, Oncogynecology, Almaty, Kazakhstan; ${ }^{2}$ Kazakh Institute of Oncology and Radiology, Head of Kazior, Almaty, Kazakhstan; ${ }^{3}$ Almaty Oncologycal Center, Oncogynecology, Almaty, Kazakhstan

\subsection{6/ijgc-2021-IGCS.103}

Objectives To investigate pregnancy outcomes in women after radical trachelectomy (RT) in KazIOR for early-stage cervical cancer

Methods Systematic analysis of the data of the cancer register of the Republic of Kazakhstan

Results Since 2013, radical trachelectomy has been performed at KazIOR. From 2013 to 2021, 8 operations were performed, 7 of them by abdominal access, 3 by laparoscopic approach. $6(75 \%)$ of the patients had stage 1 B1 from 2 to $4 \mathrm{~cm} ; 2$ (25\%) had a 1a1 stage.The average age of patients was 28 years (from 26 to 37 years). 5 (62.5\%) patients were nulliparous, 2 patients had 2 children, 1 patient had 1 child.

LVSI was negative in preoperative histological examination, and resection margins were also negative. The histological form of the tumor in all cases was squamous cell carcinoma. On average, 11 lymph nodes were removed. In 1 patient (12.5\%) after histological examination LVSI was positive, in 7 it was negative. None of the patients had metastases to the pelvic lymph nodes. During express histology, the resection margins were negative in all patients. Patients in the postoperative period were not prescribed chemoradiation therapy. of the 8 patients who retained fertility, there were 5 pregnancies, 2 miscarriages at 9-10 weeks, and 3 deliveries at 36-37 weeks of gestation.

Conclusions Thus, in 2013-2021, 8 radical trachelectomy operations were successfully performed. The data presented in this publication demonstrate that patients with stage IB1 tumors ranging in size from 2 to $4 \mathrm{~cm}$ and with favorable histology are acceptable candidates for attempted radical trachelectomy.

\section{EPV036/\#142 EUROPEAN NETWORK FOR GYNAECOLOGICAL ONCOLOGICAL TRIAL (ENGOT)-CX11/ GYNECOLOGIC ONCOLOGY GROUP (GOG) 3047I KEYNOTE-A18: PHASE 3 TRIAL OF PEMBROLIZUMAB PLUS CHEMORADIOTHERAPY IN HIGH-RISK LOCALLY ADVANCED CERVICAL CANCER}

${ }^{1} \mathrm{D}$ Lorusso*, ${ }^{2} Y$ Xiang, ${ }^{3} \mathrm{~N}$ Colombo, ${ }^{4} \mathrm{RL}$ Coleman, ${ }^{5} \mathrm{LM}$ Randall, ${ }^{6} \mathrm{~L}$ Duska, ${ }^{7} \mathrm{~K}$ Hasegawa, ${ }^{8} \mathrm{~A}$ Nogueira-Rodrigues, ${ }^{9} \mathrm{D}$ Cibula, ${ }^{10} \mathrm{M}$ Mirza, ${ }^{11} \mathrm{~B}$ You, ${ }^{12} \mathrm{~A}$ Oaknin, ${ }^{13} \mathrm{M}$ Christiaens, ${ }^{14} \mathrm{C}$ Taskiran, ${ }^{15} \mathrm{~J}$ Sehouli, ${ }^{16} \mathrm{~J}$ Korach, ${ }^{17} \mathrm{C}$ Marth, ${ }^{18} \mathrm{~K}$ Yamada, ${ }^{18} \mathrm{M}$ Puglisi, ${ }^{19} \mathrm{~S}$ Pignata. ${ }^{1}$ Associate Professor of Obstetrics and Gynecology, Catholic University of Sacred Heart, Clinical Research Development Unit Fondazione Policlinico Gemelli Irccs, Rome, Italy; ${ }^{2}$ Peking Union Medical College Hospital, Chinese Academy of Medical Sciences, Department of Gynecology and Obstetrics, Beijing, China; ${ }^{3}$ Instituto Europeo di Oncologia, Department of Gynecology, Milan, Italy; ${ }^{4}$ University of Texas MD anderson Cancer Center, Department of Gynecologic Oncology and Reproductive Medicine, Houston, USA; ${ }^{5}$ Massey Cancer Center, Virginia Commonwealth University, Department of Obstetrics and Gynecology, Richmond, USA; ${ }^{6}$ University of Virginia, Department of Obstetrics and Gynecology, Charlottesville, USA; 'Saitama Medical University, Hidaka, Department of Gynecologic Oncology, Saitama Prefecture, Japan; ${ }^{8}$ Universidade Federal de Minas Gerais, Belo Horizonte, Departamento De Clínica Médica, Minas Gerais, Brazil; ${ }^{9}$ General Faculty Hospital in Prague, First Faculty of Medicine, Charles University, Department of Obstetrics and Gynecology, Prague, Czech Republic; ${ }^{10}$ Copenhagen University Hospital, Department of Oncology, Copenhagen, Denmark; ${ }^{11} \mathrm{CITOHL}, I C-H C L$, Hospices Civils de Lyon, Université Claude Bernard Lyon 1, Department of Medical Oncology, Lyon, France; ${ }^{12}$ Vall d'Hebron Institute of Oncology (VHIO), Hospital Universitari Vall d'Hebron, Vall d'Hebron Barcelona Hospital Campus, Department of Medical Oncology, Barcelona, Spain; ${ }^{13}$ Universitair Ziekenhuis Leuven, Department of Radiation Oncology, Leuven, Belgium; ${ }^{14}$ Professor Koc University School of Medicine and VKV American Hospital, Department of Obstetrics and Gynecology, Division of Gynecologic Oncology and Turkish Society of Gynecologic Oncology, Istanbul, Turkey; ${ }^{15}$ Charité-Universitätsmedizin Berlin, Deparment of Gynecology, Berlin, Germany; ${ }^{16}$ Sheba Medical Center, Gynecology Oncology Department, Ramat Gan, Israel; ${ }^{17}$ Medizinische Universität Innsbruck, Department of Obstetrics and Gynecology, Innsbruck, Austria; ${ }^{18}$ Merck and Co., Inc., Clinical Development, Kenilworth, USA; ${ }^{19}$ Instituto Nazionale Tumori IRCCS Fondazione G Pascale, Department of Uro-gynaecological Oncology, Napoli, Italy

\subsection{6/ijgc-2021-IGCS.104}

Objectives High-risk locally advanced cervical cancer has a poor prognosis. External beam radiotherapy (EBRT) with concurrent chemotherapy followed by brachytherapy is the standard of care. The immunostimulatory activity of pembrolizumab may be enhanced by concurrent chemoradiotherapy (CCRT). Pembrolizumab monotherapy is approved for patients with PD-L1-positive recurrent or metastatic cervical cancer that progressed during or after chemotherapy. The phase 3 ENGOT-cx11/GOG 3047/KEYNOTE-A18 (NCT04221945) study is evaluating pembrolizumab with CCRT in patients with locally advanced cervical cancer.

Methods $\sim 980$ patients with high-risk (FIGO 2014 stage IB2IIB with node-positive disease or stage III-IVA), locally 
advanced, previously untreated cervical cancer will be randomized 1:1 to receive either 5 cycles of pembrolizumab $200 \mathrm{mg}$ Q3W plus CCRT followed by 15 cycles of pembrolizumab $400 \mathrm{mg}$ Q6W or 5 cycles of placebo Q3W plus CCRT followed by 15 cycles of placebo Q6W. CCRT includes 5 cycles (optional 6th dose) of cisplatin $40 \mathrm{mg} / \mathrm{m}^{2}$ Q1W plus EBRT followed by brachytherapy. Randomization is stratified by planned EBRT type (intensity-modulated radiotherapy [IMRT] or volumetric-modulated arc therapy [VMAT] vs non-IMRT or non-VMAT), screening cancer stage (IB2-IIB vs III-IVA), and planned total radiotherapy dose. Treatment will continue for 20 cycles or disease progression, unacceptable toxicity, or withdrawal. Primary endpoints are PFS per RECIST v1.1 by investigator and OS. Secondary endpoints include PFS by BICR, PFS at 2 years, OS at 3 years, CR at 12 weeks, ORR, OS and PFS by PD-L1 status, QoL, and safety. Enrollment began May 2020 and is planned for 193 sites in 30 countries. Results Not applicable

Conclusions Not applicable

\section{EPV037/\#157 INCIDENCE OF CERVICAL CANCER AND THE HPV VACCINE IN THE UNITED STATES: ARE WE SEEING RESULTS OF VACCINATION EFFORTS?}

${ }^{1} \mathrm{~A}$ Francoeur, ${ }^{2} \mathrm{C}-\mathrm{I}$ Liao, ${ }^{1} \mathrm{D}$ Wong, ${ }^{3} \mathrm{~A}$ Mann, ${ }^{4} \mathrm{MA}$ Caesar, ${ }^{5} \mathrm{~A}$ Chan, ${ }^{6} \mathrm{~B}$ Monk, ${ }^{7} \mathrm{D}$ Kapp, ${ }^{8} \mathrm{~J}$ Chan. 'University of California Los Angeles, Obstetrics and Gynecology, Los Angeles, USA; ${ }^{2}$ Kaohsiung Veterans General Hospital, Obstetrics and Gynecology, Kaohsiung City, Taiwan; ${ }^{3} \mathrm{Pal}$ Alto Medical Foundation, Research Institute, Palo Alto, USA; ${ }^{4}$ California Pacific Medical Center, Research Institute, San Francisco, USA; ${ }^{5}$ Palo Alto Medical Foundation Research Intitute, Obstetrics and Gynecology, Palo Alto, USA; ${ }^{6}$ Arizona Oncology, Gynecologic Oncology, Obstetrics and Gynecology, Phoenix, USA; ${ }^{7}$ Stanford University School of Medicine, Department of Radiation Oncology, Stanford, USA; ${ }^{8}$ California Pacific Medical Center, Obstetrics and Gynecology, San Francisco, USA

\subsection{6/ijgc-2021-IGCS.105}

Objectives To determine the incidence and trends of cervical cancer in the United States in relation to the HPV vaccine.

Methods Data were obtained from the U.S. Cancer Statistics program from 2001-2017. SEER*Stat 8.3.8 and Joinpoint regression program 4.8.0.1 were used to calculate incidence trends.

Results Over the last 17 years, cervical cancer incidence is decreasing at an average annual percent change (AAPC) of $1.03 \%(\mathrm{p}<0.001)$. We performed a subset analysis of women who were 9-13 years old in 2006 when the HPV vaccine was approved, now 20-24 years old in 2017. In the pre-vaccine era (2001-2011), the incidence of cancer decreased $2.3 \%$ annually $(p=0.038)$. of note, after the introduction of the vaccine $(2011-2017)$, it decreased at $9.6 \%$ per year $(p=0.002)$. In the pre-vaccine era (2001-2012), the incidence of new diagnoses of squamous cell carcinoma observed a decrease of $3.1 \%$ annually $(\mathrm{p}=0.004)$. However, in the post-vaccine era (2012-2017), there was an $11.8 \%$ decline in new cases per year $(p=0.007)$. Although there is a decrease in older age groups, there is no difference in the trends pre and post vaccine era, particularly in the age groups who were not eligible for vaccination at that time.

Conclusions In our population analysis, our data suggest that the HPV vaccination may have decreased in incidence of cervical cancer in the younger cohort after its approval.
EPV038/\#163 NEUROTROPHIC TYROSINE KINASE RECEPTOR-1 (NTRK-1) REARRANGED CERVICAL SARCOMA WITH FIBROSARCOMA LIKE MORPHOLOGY PRESENTING IN A 13-YEAR-OLD MANAGED WITH A NEO-ADJUVANT TRK-INHIBITOR AND SURGICAL EXCISION

${ }^{1} \mathrm{E}$ Goulding*, ${ }^{2} \mathrm{P}$ Morreau, ${ }^{3} \mathrm{M}$ De Silva, ${ }^{4} \mathrm{M}$ Watson, ${ }^{5} \mathrm{~B}$ Leung, ${ }^{6} \mathrm{C}$ Van Vliet, ${ }^{1} \mathrm{~L}$ Eva. ${ }^{1}$ National Women's Health, Gynaecologic Oncology, Auckland, New Zealand; '2Starship Children's Hospital, Paediatric Surgery, Auckland, New Zealand; '3tarship Children's Hospital, Medical Oncology, Auckland, New Zealand; ${ }^{4}$ Auckland City Hospital, Anatomical Pathology, Auckland, New Zealand; ${ }^{5}$ Starship Children's Hospital, Radiology, Auckland, New Zealand; ${ }^{6}$ QEIl Medical Centre, Anatomical Pathology, Perth, Australia

\subsection{6/ijgc-2021-IGCS. 106}

Objectives Fibrosarcoma like tumours of the uterine cervix affecting premenopausal women with neurotrophic tyrosine kinase receptor (NTRK) gene rearrangements have recently been described in the literature. They are rare tumours and to our knowledge there are only 18 cases reported, none of which has occurred in the paediatric population. We describe the first case of a paediatric patient with a NTRK fusion positive fibrosarcoma-like tumour of the uterine cervix who was successfully managed with neo-adjuvant entrectinib and subsequently went on to have conservative, fertility sparing surgery. Methods This case report reviews the case of a 13-year-old patient who presented with a $9 \mathrm{~cm}$ NTRK-1 rearranged cervical sarcoma with fibrosarcoma like morphology. At presentation the lesion filled her vagina and pelvis and any attempt at surgical removal would have been morbid and led to loss of fertility.

Results Based upon evidence that has shown good tolerability and responses of paediatric solid tumours with NTRK gene fusions to NTRK inhibitors, both in the neoadjuvant and upfront setting, this patient was managed with neo-adjuvant entrectinib. Following a dramatic reduction in tumour size confirmed by imaging, she underwent conservative fertility sparing surgery with final histopathology showing no residual disease.

Conclusions This case highlights the importance of the investigation of NTRK fusions in fibrosarcoma like tumours of the uterine cervix, as this may open up treatment options for patients and avoids potentially morbid extensive surgery, which may impair fertility.

\section{EPV039/\#175 RELATIVE IMPORTANCE OF INDIVIDUAL INSURANCE STATUS AND HOSPITAL PAYER MIX ON SURVIVAL FOR WOMEN WITH CERVICAL CANCER}

${ }^{1} \mathrm{C}$ Cherston*, ${ }^{1} Y$ Huang, ${ }^{1} \mathrm{~A}$ Melamed, ${ }^{2} \mathrm{~V}$ Prabhu, ${ }^{2} \mathrm{Y}$ Li, ${ }^{1} \mathrm{~J}$ Wright. ${ }^{1}$ Columbia University, Gynecologic Oncology, New York, USA; ${ }^{2}$ Merck and Co., Inc., Outcomes Research, Kenilworth, USA

\subsection{6/ijgc-2021-IGCS. 107}

Objectives Safety-net hospitals (SNH) are important sites of care especially for vulnerable groups (e.g., uninsured/Medicaid). We examined the relative contributions of individual insurance status and hospital payer mix on quality of care and survival for women with cervical cancer. 